\title{
Review: Analysis and Manufacturing of Ankle Foot Orthosis for Foot Drop
}

\author{
A. A. Pawale ${ }^{1}$, V. N. Chougule ${ }^{1}$, W. N. Tamboli ${ }^{1}$, A. V. Mulay ${ }^{2}$ \\ 1(Department of Mechanical Engineering, M.E.S.College of Engineering, S.P. Pune University, India) \\ 2(Department of Production \& Industrial Management, College of Engineering, Pune,, India)
}

\begin{abstract}
An Ankle Foot Orthosis is a device that supports the ankle and foot area of the body and extends from below the knee and including foot area. This device is used to control instabilities in the lower limb by maintaining proper alignment and controlling motion of the foot. Finite element analysis of models was developed to predict the mechanical behavior of AFO. The Carbon Fiber is best suitable material for manufacturing of $A F O$ also CF-AFO is capable of taking maximum stress than thermoplastic material also CF$A F O$ is light weight and durable than other materials. This is validated by conducting experimental testing and analysis by Ansys.
\end{abstract}

Keywords: $A F O$, drop foot, $C F-A F O$, Ansys, Finite element analysis.

\section{Introduction}

There are millions of individuals with gait disabilities, requiring either rehabilitation or permanent assistance. In the US alone, approximately 866,000 people use an orthosis on a lower extremity (US DoC, 1994). An orthosis is externally applied device used to modify the structural and functional characteristics of the neuromuscular and skeletal system[1]. Orthosis is used to Control and guide body segment for a particular reason also restrict movement in a given direction, reduce weight bearing forces for a particular purpose, aid rehabilitation from fractures after the removal of a cast.

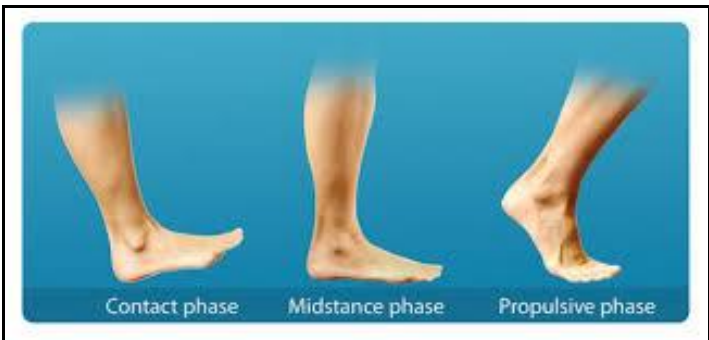

Fig.1 (a) Gait cycle of Person without foot drop.

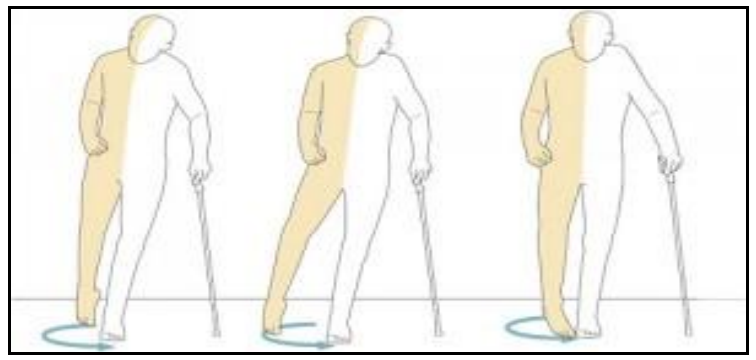

(b) Gait cycle of person with foot drop (Biomechanics)

Foot drop is a gait abnormality in which the dropping of the forefoot happens due to weakness, irritation or damage to the common nerve or paralysis of the muscles in the anterior portion of the lower leg. [2]

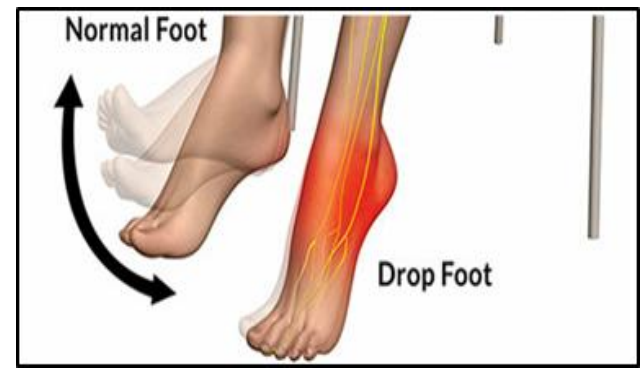

Fig.2 (a) Drop Foot [2]

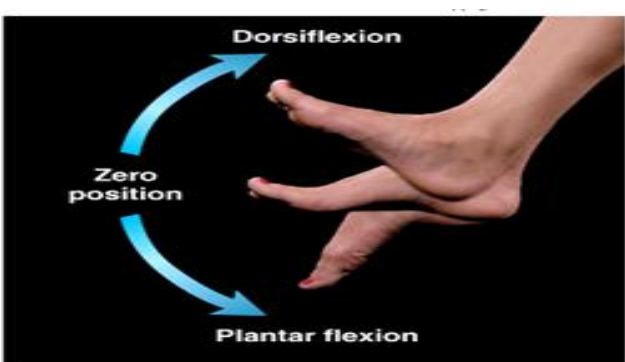

(b) Ankle Moment [2] 

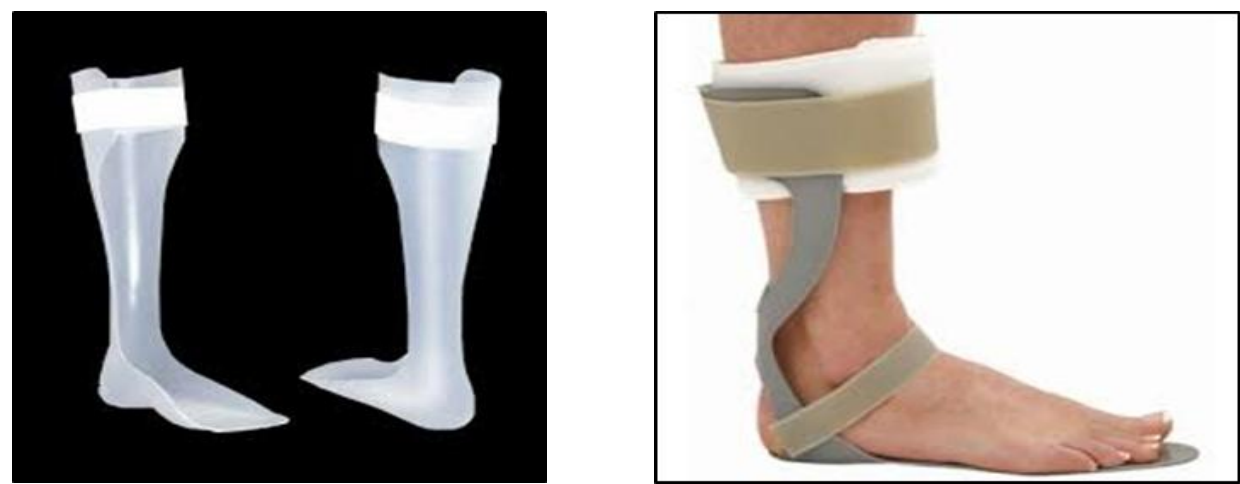

Fig.3 Patient with AFO [2]

ANKLE-FOOT ORTHOSIS (AFO) are devices often prescribed to improve gait performance for persons suffering through foot drop.[1] Drop foot, which afflicts over 300,000 people in the US alone (Nat'1 Stroke Assoc. 1993), is caused by a weakness in the dorsiflexor muscles that lift the foot. This is caused by stroke, cerebral palsy (CP)[3], myelomeningocele [5], multiple sclerosis (MS) (Taylor et al., 1999), and neurological trauma, such as accidents or surgical complications. To find solution on this they apply ankle biomechanics, engineering principles and current robotic technology to develop an improved, adaptive and autonomous system to assist drop foot gait.Motivation-The major motivation behind this researcher effort is to assist those individuals with gait disabilities. The goal is to build an adaptive assistive device for any walking disability. This "bio-suit" would be able to detect and assist any abnormal gait from a spastic ankle to paraplegia. It would also be able to provide the proper rehabilitation or permanent assistance depending on the need of the user.

\section{Material And Experimental Set Up}

Dequan Zou et.al[4] take one experimental analysis they take 2 materials one is of Carbon Fibre and second is polypropylene. They take bench top testing setup CF-AFO is loaded $1000 \mathrm{~N}$ while polypropylene AFO was loaded with $150 \mathrm{~N}$. multiple trials run on each AFO with rate of loading $8 \mathrm{~mm} / \mathrm{sec}$. and collect data. The result of CF-AFO is better than polypropylene. They compare experimental and software data and select the CF- AFO. Also Sebastian et.al uses the Carbon Fibre for AFO because it offers high elasticity, also low weight ,high strength, high stiffness, low thermal expansion ,high chemical resistance because of all this property of Carbon Fibre they uses the CF as AFO material.

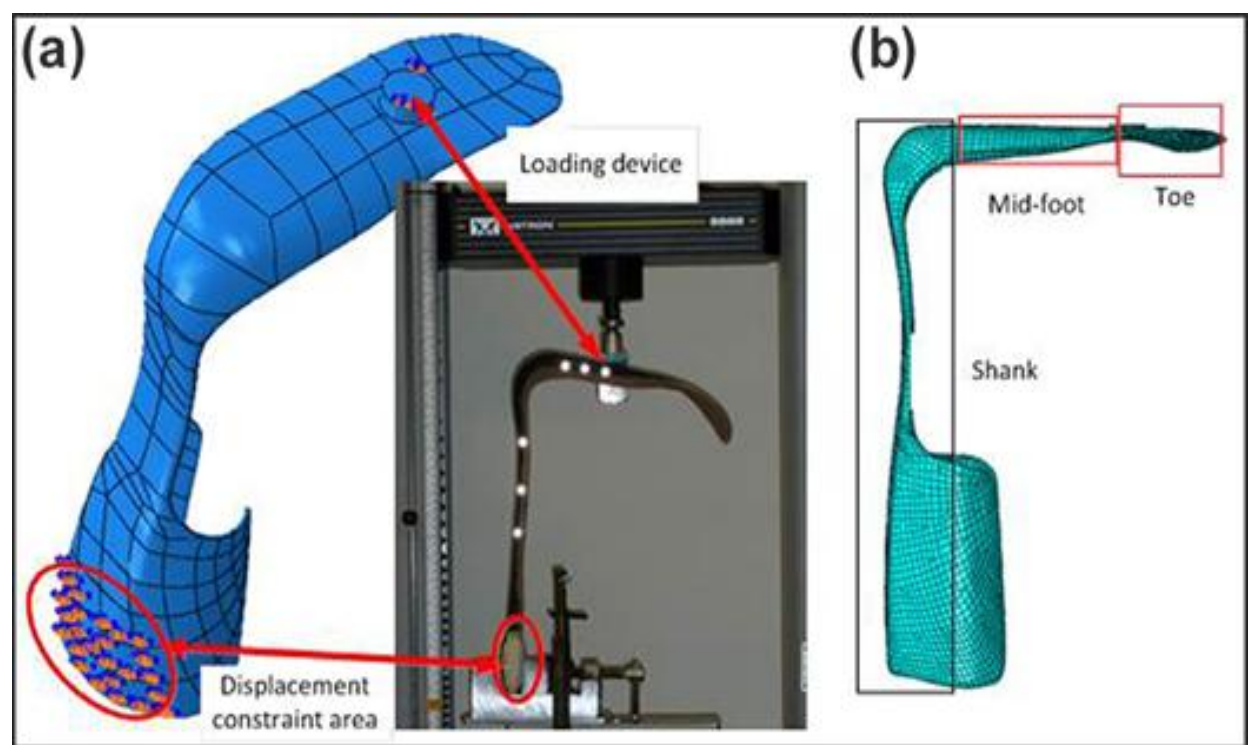

Fig.4 (a) Bench- top testing setup. (b) Finite element analysis modeling setting. [4] 


\section{Finite Element Method}

In FEA model CF- AFO was divided into three parts (shank, mid-foot, and toe). Then to make correct meshing of the object otherwise it may causes the failure of the object. The element type used a quadratic geometric order with the improved surface stress formulation. He get 276 faces of the geometry were seeded by 74,568 elements during the meshing process before simulations were run.[4] he uses the shell type element for FEA of carbon fibre and resins. Therefore stress in resins and carbon fibre we get because now load is on both material that is carbon fibre and resins.by applying load up to $1000 \mathrm{~N}$ the CF-AFO get best result from energy return point of view also and also maximum stress and load it can carry when we use CF-AFO. Dequan zou et. al[4] get the CF AFOs used in this study were able to return more than 88 percent of energy from a 1,000 N load. The thermoplastic AFO returned 77.4 percent of a $150 \mathrm{~N}$ load, deformed more than CF AFOs. Also TCF AFO is energy storage device that may be well suited to assist in the push-off phase of walking as well as preventing foot drop during the swing phase.[4][5].Hence by FEM method they get the best result from CFAFO than thermoplastics that is polypropylene in the energy return point also and maximum load carrying capacity also.[4][5][6] Also for stress distribution in ankle foot orthosis a quasi-static 3-D finite element analysis of AFO is carried out and from this analysis they find out the maximum peak stress in the region of heel, neck, and side-arc region of AFO.[7] The maximum stress is act on toe region during the push-off time when person is walking with normal gait cycle this is find out by 3-D analysis of barefoot by Weng-Pin Chen et.al.[8]

\section{Manufacturing Technique}

The 3D-printing technique is ideal for fabrication of CF-AFOs. Fused deposition modelling (FDM) is 3D-printing method HAVING desired strength and material deposition rate for CF- AFO applications. In FDM method the process planning is main thing to focus for the cycle time of Four steps in the process planning are: 1) orientation determination in this they take several factors such as mechanical performance of CF-AFO to improve the printability. 2) In support generation they focus on optimization of supporting structures. 3) Slicing strategy is used to slice the AFO with full consideration of its geometric characteristics. 4) Tool path generation the pattern is decide in the form of wavy or zigzag pattern depending on their demand during manufacturing.[2]

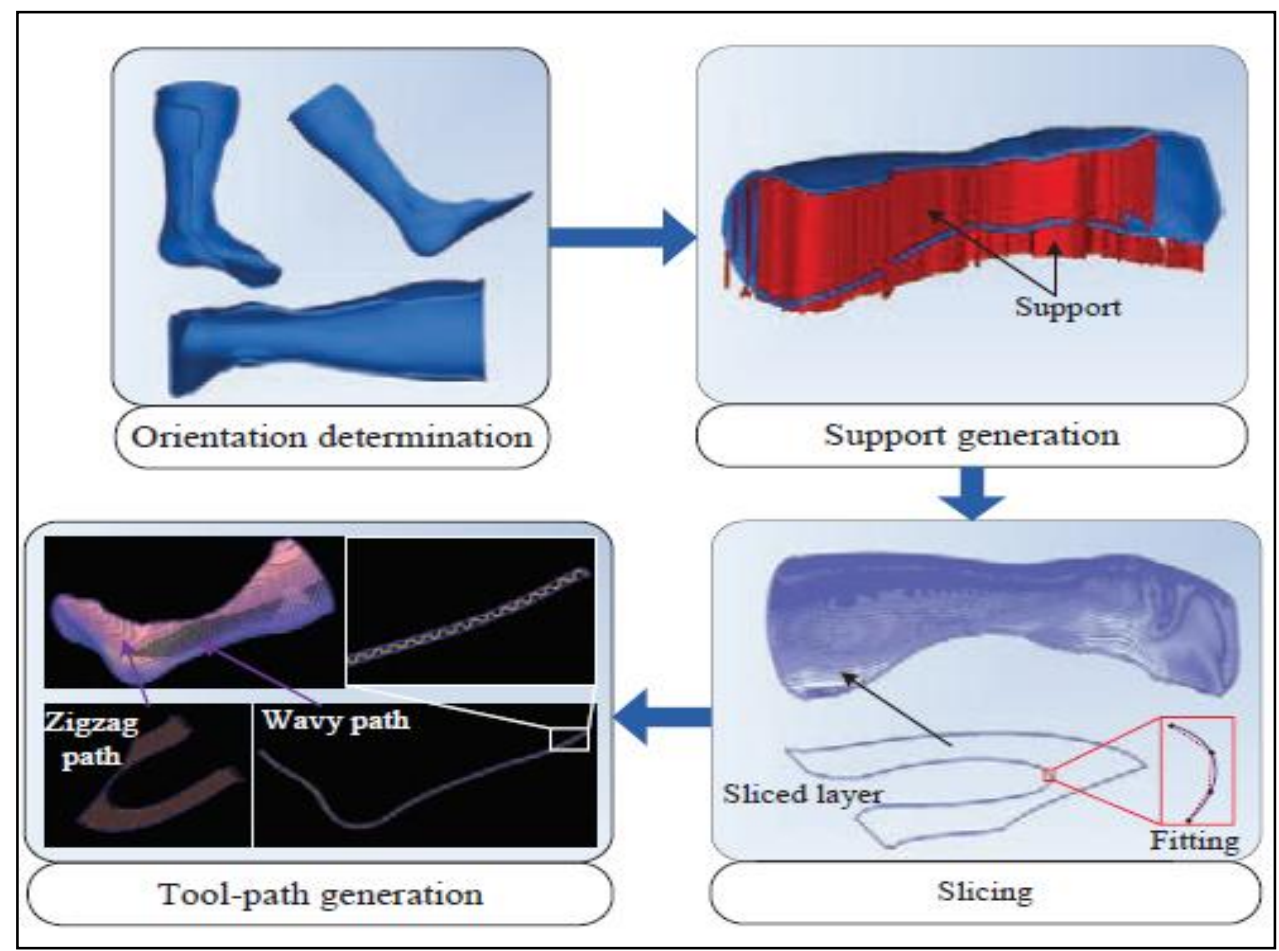

Fig.5

step process planning for 3-D PRINTING [2]

\section{Conclusion}

This study provided an overview of Ankle Foot Orthosis. From this we get clear idea to manufacture CF-AFO for person suffering from disabilities in lower limb portion. Also get the idea of finite element analysis for finding the maximum stress and displacement area and optimized this in future work and manufacturing 
techniques such as 3-D printing method and also one option is there that is custom oriented manufacturing techniques for CF-AFO. Hence from all above study we decide to manufacture CF-AFO for a person who suffers from foot drop. And also Make In India as this CF-AFO is imported from other country.

\section{Acknowledgement}

This research is supported by Dr. S.K.JAIN Surgeon in Sancheti Hospital, PUNE. For their guidance in ankle foot orthosis for the application of foot drop.

\section{References}

[1]. M. C. Faustini, Richard R. Neptune, R. H. Crawford,S. J. Stanhope, Manufacture of Passive Dynamic Ankle-Foot Orthoses Using Selective Laser Sintering, IEEE Transactions on Biomedical Engineering,55(2), 2008.

[2]. Y. Jina,b , Yong He, Albert Shih Process Planning for the Fuse Deposition Modeling of Ankle-Foot-Othoses ,Procedia CIRP $42,2016,760-765$.

[3]. J. Romkes, Reinald Brunner Comparison of a dynamic and a hinged ankle-foot orthosis by gait analysis in patients with hemiplegic cerebral palsy Gait and Posture $15,2002,18-24$

[4]. D. Zou, DS, Tao He, MS,Michael Dailey, Kirk E. Smith, M.J. Silva, D. R.Sinacore,M. J. Mueller, M.K. Hastings, Experimental and computational analysis of composite ankle foot orthosis JRRD, 51, (10), 2014.

[5]. S. I. Wolf , M. Alimusaj, O. Rettig, L.Do“derlein ,Dynamic assist by carbon fiber spring AFOs for patients with myelomeningocele Gait \& Posture, 28 ,2008, 175-177.

[6]. J. Romkes *, A.K. Hell, R. Brunner Changes in muscle activity in children with hemiplegic cerebral palsy while walking with and without ankle-foot orthoses Gait \& Posture 24,2006,467-474

[7]. T.Ming Chu, N. P. Reddy,Stress distribution in the ankle-foot orthosis used to correct pathological gait, Journal of Rehabilitation Research and Development,32(4), 1995,349-360

[8]. W.pin Chen, Fuk-Tan Tang, Chia-wei, Ju Stress distribution of foot during mid- stance to push-off in barefoot gait-a 3-D finite element analysis,.Clinical Biomechanics ,16,2001,614-620. 